Research Paper

\title{
Role of Adjuvant Chemotherapy in Advanced Stage Upper Urinary Tract Urothelial Carcinoma after Radical Nephroureterectomy: Competing Risk Analysis after Propensity Score-Matching
}

\author{
Minyong Kang ${ }^{1,2}$, Heejin Yoo $^{3}$, Kyunga Kim³ ${ }^{3}$ Si Hyun Sung${ }^{1}$, Hwang Gyun Jeon¹, Se Hoon Park ${ }^{4}$, Seong Il \\ Seo ${ }^{1}$, Seong Soo Jeon ${ }^{1}$, Hyun Moo Lee ${ }^{1}$, Han Yong Choi ${ }^{5}$ and Byong Chang Jeong ${ }^{\circledR}$ \\ 1. Department of Urology, Samsung Medical Center, Sungkyunkwan University School of Medicine \\ 2. Department of Health Sciences and Technology, SAIHST, Sungkyunkwan University; \\ 3. Statistics and Data Center, Samsung Medical Center; \\ 4. Division of Hematology-Oncology, Department of Internal Medicine, Samsung Medical Center, Sungkyunkwan University School of Medicine; \\ 5. Department of Urology, Kangbuk Samsung Hospital, Seoul, Republic of Korea. \\ $\square$ Corresponding author: Byong Chang Jeong, M.D., Ph.D. Department of Urology, Samsung Medical Center, Sungkyunkwan University School of Medicine, 81 \\ Irwon-Ro, Gangnam-gu, Seoul 06351, Republic of Korea, E-mail: bc2.jung@samsung.com; Tel: +82-2-3410-3557
}

(1) The author(s). This is an open access article distributed under the terms of the Creative Commons Attribution License (https://creativecommons.org/licenses/by/4.0/). See http://ivyspring.com/terms for full terms and conditions.

Received: 2019.02.14; Accepted: 2019.09.14; Published: 2019.11.17

\begin{abstract}
Objective: To determine whether adjuvant chemotherapy $(\mathrm{ACH})$ influences cancer-specific mortality, bladder cancer-specific mortality, and other-cause mortality in patients with locally advanced upper tract urothelial carcinoma (UTUC) following radical nephroureterectomy (RNU) through the use of competing risk analysis.
\end{abstract}

Methods: Among 785 patients with UTUC who underwent RNU from 1994 through 2015, we analyzed 338 individuals with locally advanced UTUC (pathologic T3-T4 and/or positive lymph nodes) without distant metastases. Patients were classified into two groups according to receipt of $\mathrm{ACH}$. We performed a 1:1 propensity score-matching analysis between the $\mathrm{ACH}$ and no $\mathrm{ACH}$ group. The study endpoints were UTUC- and other cause-specific survivals. The association of potential risk factors with outcome was tested with the Fine and Gray regression model.

Results: During a median follow-up duration of 31.5 months, rates of UTUC- and other cause-mortalities were $32.9 \%(n=79)$ and $8.7 \%(n=21)$, respectively. Of note, there were no significant differences in overall survival between the observation and $\mathrm{ACH}$ groups according to the competing risks of death (UTUC and other causes of death). Multivariate analysis showed that only older age at surgery ( $\geq 65$ years; hazard ratio $[H R]=1.73)$, multifocality $(H R=1.74)$, and tumor size $(H R=1.92)$ remained as poor predictors of UTUC-specific survival. Additionally, positive surgical margin was only identified as independent predictor of other causes of death $(H R=4.23)$.

Conclusion: In summary, postoperative chemotherapy failed to improve UTUC- and other cause-specific survival rates, based on competing risk analysis after propensity score-matching.

Key words: Upper urinary tract cancer; Locally-advanced; Adjuvant chemotherapy; Competing risk analysis; Propensity score-matching.

\section{Introduction}

Upper urinary tract urothelial carcinoma (UTUC) is a rare and aggressive disease associated with high morbidity and mortality [1]. Radical nephroureterectomy (RNU) is the mainstay of treatment for non-metastatic UTUC [2]. However, the prognosis of patients with advanced stage UTUC, such as $\geq$ pathologic T3 (pT3) or node positive [pN $(+)$ ] disease, has not changed over the past decades 
[3]. Patients with UTUC are diagnosed with locally advanced, high-grade disease following surgery should be considered for administration of adjuvant chemotherapy (ACH) [4]. Although European Association of Urology (EAU) guidelines state that cisplatin-based $\mathrm{ACH}$ can exert a beneficial effect on both overall survival (OS) and disease-free survival, the current practice is mainly dependent on data from bladder cancer [2,5-7]. Thus, a definitive recommendation is unlikely until high-quality evidence from randomized prospective trials is obtained in patients with UTUC. Moreover, retrospective studies have shown conflicting results regarding the effects of $\mathrm{ACH}$ on oncological outcomes in patients with UTUC [8-11]. In this regard, the role of $\mathrm{ACH}$ for patients with advanced stage UTUC remains an open question.

Because patients with UTUC are at high risk of intravesical recurrence, as well as renal insufficiency, following surgery and subsequent $\mathrm{ACH}$, traditional methods of survival estimation, such as Kaplan Meier analysis or Cox proportional hazards modeling, may be inappropriate to evaluate the competing nature of these multiple causes of mortality. Competing risk analysis is a novel method of survival analysis that aims to correctly predict the marginal probability of a specific event in the presence of competing causes, providing more accurate information regarding the multiple competing events [12]. However, to the best of our knowledge, there have been no reports on the role of $\mathrm{ACH}$ in patients with advanced stage UTUC that have utilized competing risk analysis. In the present study, we investigate the influence of $\mathrm{ACH}$ on survival outcomes in patients with advanced UTUC following RNU, particularly based on competing risk analysis after propensity score-matching for the first time.

\section{Patients and Methods}

\section{Study population}

We retrospectively reviewed clinical data from 785 patients with UTUC who underwent RNU between September 1994 and December 2015 at Samsung Medical Center. After excluding 447 patients with localized disease, we finally analyzed 338 patients with locally advanced UTUC [pathologic T3-T4 (pT3-4) and/or positive lymph nodes ( $\mathrm{pN}(+))]$ without distant metastase $(\mathrm{n}=265$ [pT3-4], $\mathrm{n}=73$ [pN $(+)])$. We evaluated the following clinicopathological parameters: age at surgery, sex, body mass index (BMI), preoperative use of ureteroscopy, type of operation (open or laparoscopic surgery), tumor laterality, tumor size, tumor location, pathologic $\mathrm{T}$ and $\mathrm{N}$ stage, tumor grade, presence of lymphovascular invasion (LVI), surgical margin status, multifocality, bladder cuffing type, and receipt of adjuvant systemic chemotherapy (ACH).

The Institutional Review Board (IRB) at our hospital approved the current study (SMC 201804-011-001). Because this study was retrospectively performed, the IRB waived the requirement to document informed consent from the included patients. All procedures performed in the present study were in accordance with the Declaration of Helsinki guidelines.

\section{Study design}

RNU with bladder cuff excision was performed according to the surgical protocol described previously [13]. The type of surgery, open or laparoscopic RNU, was determined at the attending surgeons' discretion. Bladder cuff excision was typically conducted via the extravesical technique with a modified Gibson incision, by removing the entire ureter including the ureteral orifice. In cases of evidence of clinically significant lymph node enlargement on preoperative images and/or direct examination during surgery, we performed retroperitoneal lymph node dissection. Surgical tissue specimens were placed into $10 \%$ formalin solution for fixation and were then processed into paraffin blocks. Paraffin-embedded tissues were sectioned into 4-6 $\mu \mathrm{m}$ slices and mounted onto glass slides, followed by hematoxylin and eosin staining. Experts on genitourinary tract pathology carefully reviewed these slides and reported the key pathological findings. Pathologic T stage and tumor grade were determined by the 2009 American Joint Committee on Cancer staging system and the 1973 World Health Organization/International Society of Urologic Pathology consensus classification, respectively [14, 15]. LVI was defined as the spread of cancer cells to the blood vessels and/or lymphatics within the upper urinary tract. Positive resection margin was defined as the presence of cancer cells at the end of the distal resection site of surgical specimens.

Patients received three to six courses of $\mathrm{ACH}$ based on a gemcitabine and cisplatin regimen at one month after surgery. Patients were classified into two groups according to receipt of $\mathrm{ACH}$ after surgery. To reduce the selection bias between patients receiving $\mathrm{ACH}$ or not, we performed a 1: 1 propensity score-matching analysis. Propensity scores were estimated by using a logistic regression model of the 8 covariates (sex, age at surgery, BMI, preoperative URS, LVI, tumor location, tumor size, pathologic N stage). The primary endpoint was UTUC-specific survival, and secondary endpoints were bladder cancer- and other cause-specific survivals. The 
patients underwent routine follow-up every three months during the first two years after surgery. Patients received follow-up every six months during the third year after surgery, and then checked annually thereafter. We typically performed history-taking, physical examination, routine laboratory tests including urine cytology, cystoscopy, chest radiography, and computed tomographic (CT) urography during the follow-up periods.

\section{Statistical analysis}

We described the results of descriptive analyses of continuous variables as the median values with interquartile ranges (IQRs), as well as the actual numbers and proportions (\%) of events. We performed the Mann-Whitney $U$ test and chi-square test to identify the statistical differences in continuous and categorical variables, respectively. The association of potential risk factors with competing risk outcome was tested using the Fine and Gray regression model. Tumor size was non-normally distributed and was therefore transformed using log transformation prior to its inclusion in statistical analyses. We presented the results of statistical analysis as hazard ratios (HR) with $95 \%$ confidence intervals (CI). Statistically significant outcomes were

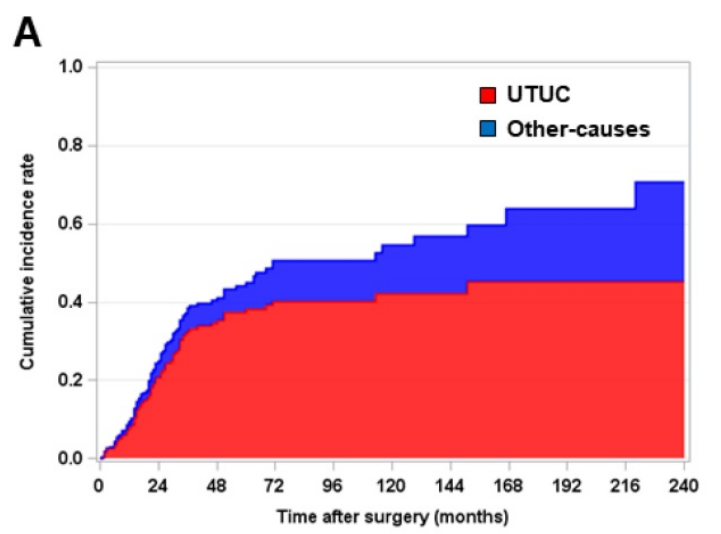

B

C

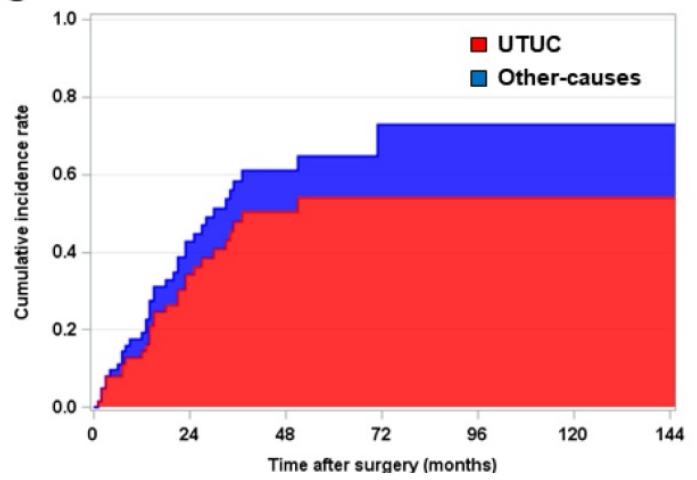

Figure 1. Kaplan-Meier survival curve analysis for estimating competing risk of death in patients with (A) pT3-4 and/or pN (+) and (B) pT3-4 and pN (-) and (C) PT any $\mathrm{N}(+)$ upper urinary tract urothelial carcinoma who underwent radical nephroureterectomy.

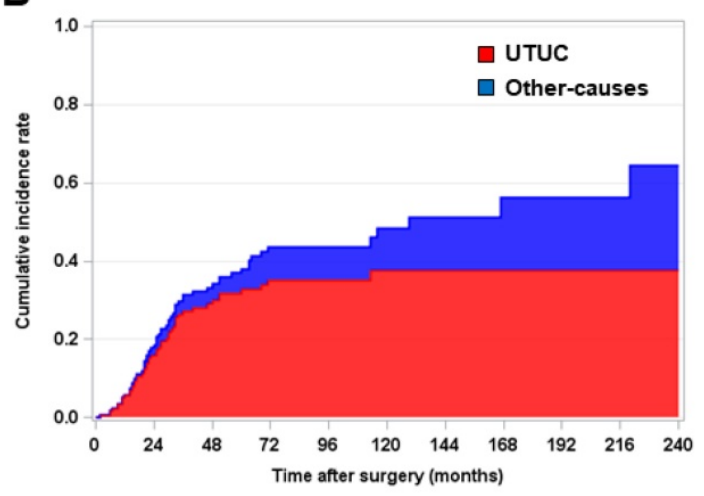

indicated by a two-sided p-value less than 0.05 . All statistical analysis in this study was executed by the Statistics and Data Center at Samsung Medical Center using SAS version 9.4 (SAS Institute Inc., Cary, NC, USA).

\section{Results}

Among the entire group of 338 patients, $42.6 \%$ (n $=144$ ) received $\mathrm{ACH}$ following RNU. To minimize the selection bias between the $\mathrm{ACH}$ and no $\mathrm{ACH}$ group, we performed a 1: 1 propensity score-matching analysis. The baseline characteristics of the pre-propensity $(n=338)$ and post-propensity $(n=240)$ populations are shown in Table 1. After propensity score-matching analysis, no variables were significantly different between the $\mathrm{ACH}$ group $(\mathrm{n}=$ 120 ) and no ACH group ( $\mathrm{n}=120)$. During a median follow-up period of 31.5 months (IQR $=16.0-65.0)$, UTUC-specific death and other-causes of death rates were $32.9 \%(n=79)$ and $8.7 \%(n=21)$, respectively. Fig. 1 shows graphic illustrations of competing risk analysis according to the cause of death, such as UTUC- and other-causes specific, in the overall, pT3-4 and $\mathrm{pN}(-)$, and $\mathrm{pT}$ any and $\mathrm{pN}(+)$ populations. 
Table 1. Baseline demographics of patients with advanced upper urinary tract carcinoma who underwent radical nephroureterectomy and adjuvant chemotherapy: Pre and post propensity score matching data.

\begin{tabular}{|c|c|c|c|c|c|c|c|c|}
\hline \multirow[t]{2}{*}{ Variables } & \multicolumn{4}{|c|}{ Pre-propensity score match } & \multicolumn{4}{|c|}{ Post-propensity score match } \\
\hline & Total & No ACH & $\mathrm{ACH}$ & P-value & Total & $\mathrm{No} A C H$ & $\mathrm{ACH}$ & P-value \\
\hline No. of patients & $338(100.0)$ & $193(57.1)$ & $145(42.9)$ & & $240(100.0)$ & $120(50.0)$ & $120(50.0)$ & \\
\hline \multicolumn{9}{|c|}{ Age at systemic therapy } \\
\hline Median (IQR) & $65(57-72)$ & $68(58-74)$ & $62(56-68)$ & $<0.001$ & $63(56-70)$ & $62(55-72)$ & $64(58-69)$ & 0.435 \\
\hline$<65$ years & $161(47.6)$ & $73(37.8)$ & $88(60.7)$ & $<0.001$ & $129(53.7)$ & $64(53.3)$ & $65(54.2)$ & 0.873 \\
\hline$\geq 65$ years & $177(52.4)$ & $120(62.2)$ & $57(39.3)$ & & $111(46.3)$ & $56(46.7)$ & $55(45.8)$ & \\
\hline \multicolumn{9}{|l|}{ Sex } \\
\hline Male & 245 (72.7) & $136(70.5)$ & $109(75.2)$ & 0.323 & $174(72.5)$ & $87(72.5)$ & $87(72.5)$ & 1.000 \\
\hline Female & $93(27.3)$ & $57(29.5)$ & $36(24.8)$ & & $66(27.5)$ & $33(27.5)$ & $33(27.5)$ & \\
\hline \multicolumn{9}{|c|}{ Body mass index $\left(\mathrm{kg} / \mathrm{m}^{2}\right)$} \\
\hline Median (IQR) & $24.1(22.3-25.9)$ & $23.8(22.0-25.5)$ & $24.3(22.8-26.3)$ & 0.064 & $24.2(22.7-26.1)$ & $24.1(22.6-26.1)$ & $24.3(22.9-26.1)$ & 0.732 \\
\hline$<25$ & $220(65.1)$ & $133(68.9)$ & $87(60.0)$ & 0.107 & $146(60.8)$ & $74(61.7)$ & $72(60.0)$ & 0.768 \\
\hline$\geq 25$ & $118(34.9)$ & $60(31.1)$ & $58(40.0)$ & & $94(39.2)$ & $46(38.3)$ & $48(40.0)$ & \\
\hline \multicolumn{9}{|l|}{ Preoperative URS } \\
\hline No & $194(57.4)$ & 119 (61.7) & $75(51.7)$ & 0.094 & $137(57.1)$ & $70(58.3)$ & $67(55.8)$ & 0.674 \\
\hline Yes & $144(42.6)$ & $74(38.3)$ & $70(48.3)$ & & 103 (42.9) & $50(41.7)$ & $53(44.2)$ & \\
\hline \multicolumn{9}{|l|}{ Operation type } \\
\hline Open & $202(59.8)$ & $117(60.6)$ & 85 (58.6) & 0.736 & $138(57.5)$ & $71(59.2)$ & $67(55.8)$ & 0.600 \\
\hline Laparoscopy & $136(40.2)$ & $76(39.4)$ & $60(41.4)$ & & $102(42.5)$ & $49(40.8)$ & $53(44.2)$ & \\
\hline \multicolumn{9}{|l|}{ Tumor laterality } \\
\hline Right & 161 (47.6) & 99 (51.3) & $62(42.8)$ & 0.152 & $111(46.2)$ & $59(49.2)$ & $52(43.3)$ & 0.392 \\
\hline Left & $177(52.4)$ & $94(48.7)$ & $83(57.2)$ & & $129(53.8)$ & $61(50.8)$ & $68(56.7)$ & \\
\hline Tumor size $(\mathrm{cm})$ & $4.0(2.7-5.8)$ & $4.0(2.7-5.8)$ & $4.0(2.8-5.2)$ & 0.751 & $4.0(2.5-5.5)$ & $4.0(2.5-5.7)$ & $4.0(2.5-5.1)$ & 0.872 \\
\hline \multicolumn{9}{|l|}{ Tumor location } \\
\hline Renal pelvis & $187(55.3)$ & $116(60.1)$ & $70(48.3)$ & & $128(53.3)$ & $66(55.0)$ & $62(51.7)$ & \\
\hline Ureter & $103(30.5)$ & $51(26.4)$ & $53(36.5)$ & 0.091 & $77(32.1)$ & $36(30.0)$ & $41(34.2)$ & 0.787 \\
\hline Both & $48(14.2)$ & $26(13.5)$ & $22(15.2)$ & & 35 (14.6) & $18(15.0)$ & $17(14.1)$ & \\
\hline \multicolumn{9}{|l|}{ Tumor grade } \\
\hline G1-2 & $108(32.0)$ & 65 (33.7) & $43(29.7)$ & 0.479 & $78(32.5)$ & $41(34.2)$ & $37(30.8)$ & 0.532 \\
\hline G3 & $230(68.0)$ & $128(66.3)$ & $102(70.3)$ & & $162(67.5)$ & $79(65.8)$ & $83(69.2)$ & \\
\hline \multicolumn{9}{|l|}{ LVI } \\
\hline Absence & $225(66.6)$ & $134(69.4)$ & $90(62.1)$ & 0.200 & $153(63.7)$ & $78(65.0)$ & $75(62.5)$ & 0.647 \\
\hline Presence & $113(33.4)$ & $59(30.6)$ & $55(37.9)$ & & $87(36.3)$ & $42(35.0)$ & 45 (37.5) & \\
\hline \multicolumn{9}{|l|}{ Resection margin } \\
\hline Negative & $318(94.1)$ & $182(94.3)$ & $136(93.8)$ & 1.000 & 224 (93.3) & $111(92.5)$ & $113(94.2)$ & 0.593 \\
\hline Positive & $20(5.9)$ & $11(5.7)$ & $9(6.2)$ & & $16(6.7)$ & $9(7.5)$ & $7(5.8)$ & \\
\hline \multicolumn{9}{|l|}{ Multifocality } \\
\hline No & $244(72.2)$ & $141(73.1)$ & $103(71.1)$ & 0.713 & $177(73.7)$ & $89(74.2)$ & $88(73.3)$ & 0.878 \\
\hline Yes & $94(27.8)$ & $52(26.9)$ & $42(28.9)$ & & $63(26.3)$ & $31(25.8)$ & $32(26.7)$ & \\
\hline \multicolumn{9}{|c|}{ Pathologic N stage } \\
\hline $\mathrm{pN}(-)$ & $265(78.4)$ & $155(80.3)$ & $110(75.9)$ & 0.350 & $178(74.2)$ & 89 (74.2) & $89(74.2)$ & 1.000 \\
\hline $\mathrm{pN}(+)$ & $73(21.6)$ & $38(19.7)$ & $35(24.1)$ & & $62(25.8)$ & $31(25.8)$ & $31(25.8)$ & \\
\hline \multicolumn{9}{|l|}{ Cause of death } \\
\hline UTUC & $110(32.6)$ & $62(32.1)$ & $48(33.3)$ & & $79(32.9)$ & $36(30.0)$ & $43(35.8)$ & \\
\hline Other causes & $31(9.2)$ & $21(10.9)$ & $10(7.0)$ & & $21(8.7)$ & $13(10.8)$ & $8(6.6)$ & \\
\hline
\end{tabular}

$\mathrm{ACH}$, adjuvant chemotherapy; IQR, interquartile ratio; URS, ureterorenoscopy; LVI, lymphovascular invasion; UTUC, upper tract urothelial carcinoma.

A

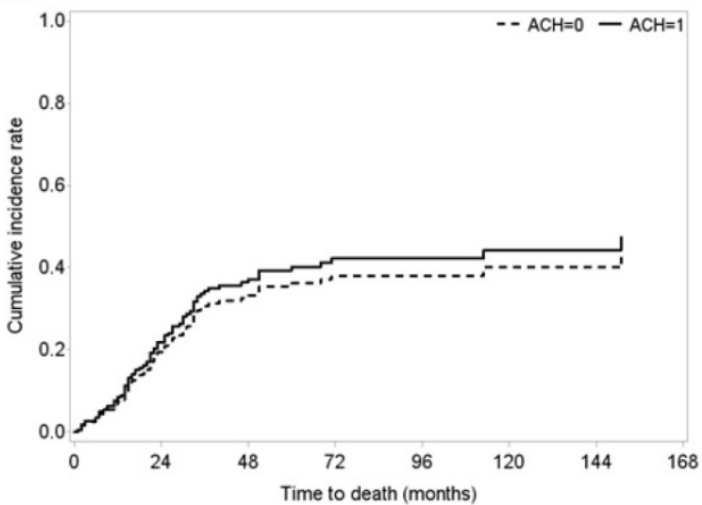

B

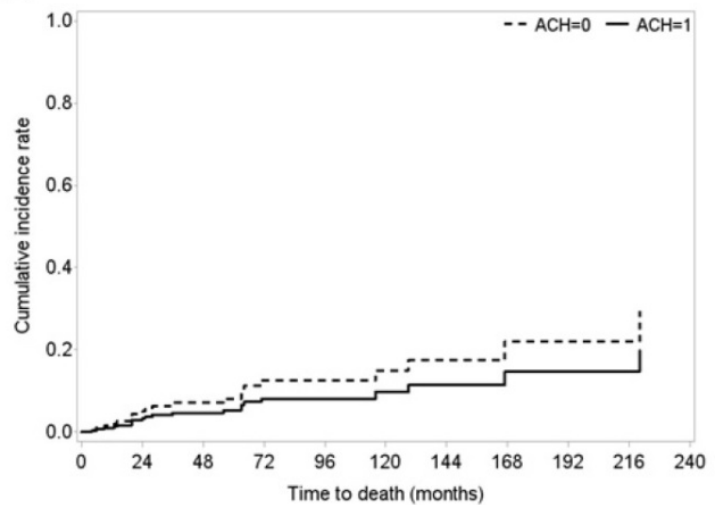

Figure 2. Cumulative incidence rates of (A) UTUC-specific death and (B) other-causes of death in patients with pT3-4 and/or pN (+) upper urinary tract urothelial carcinoma (UTUC) who underwent radical nephroureterectomy (RNU) according to receipt of adjuvant chemotherapy (ACH), using competing risk analysis. All survival analysis was performed after a 1:1 propensity score-matching between the $\mathrm{ACH}$ group and no $\mathrm{ACH}$ group.

Notably, we found no significant differences in survival outcomes between the observation and $\mathrm{ACH}$ groups in patients with $\mathrm{pT} 3-4$ and/or $\mathrm{pN}(+)$ according to the competing risks of death, UTUC and other-causes of death, respectively (Fig. 2). We also found no differences in survival outcomes between 
patients in the observation and $\mathrm{ACH}$ groups in patients with pT3-4 and $\mathrm{pN}(-)$ stage according to the competing risks of UTUC-specific and other-causes of death (Fig. S1). Finally, there were no statistical differences between patients in the observation and $\mathrm{ACH}$ groups among those with stage $\mathrm{pN}(+)$ disease, according to the competing risks of death, UTUC-specific and other-causes of death (Fig. S2).

Table 2. Multivariate analyses of predictors of cancer-specific, bladder cancer-specific and other cause-specific survival in patients with $\mathrm{pT} 3-4$ and/or $\mathrm{pN}(+)$ urothelial carcinoma of upper urinary tract.

\begin{tabular}{|c|c|c|c|c|c|c|}
\hline \multirow[t]{2}{*}{ Variables } & \multicolumn{3}{|c|}{ UTUC } & \multicolumn{3}{|c|}{ Other causes } \\
\hline & HR & $95 \% \mathrm{CI}$ & $P$ & HR & $95 \% \mathrm{CI}$ & $P$ \\
\hline \multicolumn{7}{|c|}{$\begin{array}{l}\text { Adjuvant } \\
\text { chemotherapy }\end{array}$} \\
\hline No & \multicolumn{3}{|c|}{ Reference } & \multicolumn{2}{|c|}{ Reference } & \\
\hline Yes & 1.14 & $0.72-1.80$ & 0.559 & 0.64 & $0.27-1.49$ & 0.298 \\
\hline \multicolumn{7}{|c|}{ Age at surgery } \\
\hline$<65$ years & \multicolumn{3}{|c|}{ Reference } & \multicolumn{2}{|c|}{ Reference } & \\
\hline$\geq 65$ years & 1.73 & $1.07-2.78$ & 0.023 & 1.35 & $0.54-3.40$ & 0.515 \\
\hline Tumor size & 1.92 & $1.17-3.14$ & 0.009 & 0.59 & $0.24-1.50$ & 0.274 \\
\hline \multicolumn{7}{|c|}{ Tumor grade } \\
\hline G1 - 2 & \multicolumn{3}{|c|}{ Reference } & \multicolumn{2}{|c|}{ Reference } & \\
\hline G3 & 1.27 & $0.75-2.14$ & 0.373 & 1.46 & $0.48-4.40$ & 0.505 \\
\hline \multicolumn{7}{|l|}{ LVI } \\
\hline Absence & \multicolumn{3}{|c|}{ Reference } & \multicolumn{2}{|c|}{ Reference } & \\
\hline Presence & 1.51 & $0.89-2.53$ & 0.119 & 1.18 & $0.54-2.55$ & 0.672 \\
\hline \multicolumn{7}{|l|}{ Margin } \\
\hline Negative & \multicolumn{3}{|c|}{ Reference } & \multicolumn{2}{|c|}{ Reference } & \\
\hline Positive & 0.51 & $0.16-1.61$ & 0.250 & 4.23 & $1.32-13.53$ & 0.015 \\
\hline \multicolumn{7}{|c|}{ Multifocality } \\
\hline None & \multicolumn{3}{|c|}{ Reference } & \multicolumn{2}{|c|}{ Reference } & \\
\hline Yes & 1.74 & $1.04-2.92$ & 0.035 & 1.11 & $0.41-2.98$ & 0.833 \\
\hline \multicolumn{7}{|c|}{ Pathologic N stage } \\
\hline $\mathrm{pN}(-)$ & \multicolumn{3}{|c|}{ Reference } & \multicolumn{2}{|c|}{ Reference } & \\
\hline $\mathrm{pN}(+)$ & 1.34 & $0.79-2.29$ & 0.277 & 1.81 & $0.66-4.96$ & 0.246 \\
\hline
\end{tabular}

We performed multivariate analysis using the Fine and Gray competing risks regression model to identify the predictors of survival outcomes with respect to the cause of death. In patients with stage pT3-4 and/or pN (+) UTUC, older age at surgery ( $\geq$ 65 years; $\mathrm{HR}=1.73,95 \% \mathrm{CI}=1.07-2.78)$, tumor size $(\mathrm{HR}=1.92,95 \% \mathrm{CI}=1.17-3.14)$ and multifocality (HR $=1.74,95 \% \mathrm{CI}=1.04-2.92)$ remained as poor predictors of UTUC-specific survival (Table 2). Positive resection margin $(\mathrm{HR}=4.23,95 \% \mathrm{CI}=1.32$ 13.53) was only identified as a predictive factor of other causes of death. Conversely, no statistical significance was observed for $\mathrm{ACH}$ as an independent prognosticator. In patients with stage $\mathrm{pT} 3-4$ and $\mathrm{pN}$ (-) UTUC, only older age at surgery ( $\geq 65$ years; $\mathrm{HR}=$ $1.87,95 \% \mathrm{CI}=1.08-3.23$ ) was proved as poor predictive factor for UTUC-specific survival, but no variable was identified as a predictor of other-causes of death. Similar to the results for the overall population, the use of $\mathrm{ACH}$ was not identified as a predictor for any competing risk of death (Table 3). In patients with stage $\mathrm{pN}(+)$ UTUC, we could not perform multivariate competing risk analysis owing to the small sample size $(n=44)$ (data not shown).

Table 3. Multivariate analyses of predictors of cancer-specific, bladder cancer-specific and other cause-specific survivals in patients with $\mathrm{pT} 3-4$ and $\mathrm{pN}(-)$ urothelial carcinoma of upper urinary tract.

\begin{tabular}{|c|c|c|c|c|c|c|}
\hline \multirow[t]{2}{*}{ Variables } & \multicolumn{3}{|l|}{ UTUC } & \multicolumn{3}{|c|}{ Other causes } \\
\hline & HR & $95 \% \mathrm{CI}$ & $\mathrm{P}$ & HR & $95 \% \mathrm{CI}$ & $\mathrm{P}$ \\
\hline \multicolumn{7}{|c|}{$\begin{array}{l}\text { Adjuvant } \\
\text { chemotherapy }\end{array}$} \\
\hline No & \multicolumn{3}{|l|}{ Reference } & \multicolumn{3}{|l|}{ Reference } \\
\hline Yes & 1.34 & $0.74-2.41$ & 0.325 & 0.46 & $0.14-1.44$ & 0.184 \\
\hline \multicolumn{7}{|c|}{ Age at surgery } \\
\hline$<65$ years & \multicolumn{3}{|l|}{ Reference } & \multicolumn{3}{|l|}{ Reference } \\
\hline$\geq 65$ years & 1.87 & $1.08-3.23$ & 0.025 & 0.91 & $0.28-2.96$ & 0.875 \\
\hline \multicolumn{7}{|c|}{ Tumor grade } \\
\hline G1 - 2 & \multicolumn{3}{|l|}{ Reference } & \multicolumn{3}{|l|}{ Reference } \\
\hline G3 & 1.39 & $0.76-2.54$ & 0.279 & 1.28 & $0.34-4.78$ & 0.708 \\
\hline \multicolumn{7}{|l|}{ LVI } \\
\hline Absence & \multicolumn{3}{|l|}{ Reference } & \multicolumn{3}{|l|}{ Reference } \\
\hline Presence & 1.77 & $0.97-3.21$ & 0.061 & 0.67 & $0.22-2.06$ & 0.488 \\
\hline \multicolumn{7}{|c|}{ Multifocality } \\
\hline None & \multicolumn{3}{|l|}{ Reference } & \multicolumn{3}{|l|}{ Reference } \\
\hline Yes & 1.79 & $0.91-3.55$ & 0.091 & 2.29 & 0.7407 .04 & 0.148 \\
\hline
\end{tabular}

UTUC, upper tract urothelial carcinoma; HR, hazard ratio; CI, confidence interval; LVI, lymphovascular invasion.

\section{Discussion}

Extrapolating from the data on bladder cancer and limited UTUC studies, platinum-based $\mathrm{ACH}$ is expected to be beneficial in patients with advanced stage UTUC [16]. However, there are currently insufficient data regarding the oncological role of $\mathrm{ACH}$ in patients with advanced UTUC, without level I evidence, as well as inconsistent results of retrospective studies. Necchi et al. [10] recently analyzed 1,544 patients with UTUC from a multicenter cohort and reported that no difference was observed in all-cause mortality between patients receiving $\mathrm{ACH}$ and those receiving observation following surgery. Kim et al. [8] also found no significant disease-specific or overall survival benefits associated with $\mathrm{ACH}$ in 138 patients who underwent RNU for locally advanced UTUC (pT3/4 or $\mathrm{pN}(+))$. Conversely, Seisen and colleague evaluated 3,253 individuals with $\mathrm{pT} 3 / 4$ and/or $\mathrm{pN}(+)$ who received $\mathrm{ACH}$ or observation following RNU, and found that $\mathrm{ACH}$ was significantly associated with OS benefits in both the overall population $(\mathrm{HR}=0.77)$ and all subgroups evaluated [11]. A research group in Japan performed propensity score-matched analysis in high-risk UTUC patients, and showed that there was no statistical difference in 5-year cancer-specific survival (CSS) between patients with $\mathrm{ACH}$ and those 
without $\mathrm{ACH}$ (69.0\% in the RNU and $\mathrm{ACH}$ group vs. $58.9 \%$ in the no $\mathrm{ACH}$ group [P = 0.030]) [9]. More recently, a multicenter, randomized study (the POUT trial) has been performed to prove the benefit of $\mathrm{ACH}$ following surgery in patients with locally advanced UTUC. Although there were significant improvements in disease-free survival (HR 0.49 [95\% $\mathrm{CI}=0.31-0.76]$ ) and recurrence-free survival (HR 0.49 [95\% CI $=0.30-0.78]$ ) in $\mathrm{ACH}$ group, the results are still immature to adopt as a convincing evidence of beneficial role of $\mathrm{ACH}$ in patients with UTUC. Therefore, the role of $\mathrm{ACH}$ has remained poorly defined for the management of patients with high-risk UTUC following surgery.

In the present study, we performed a competing risk analysis and first reported that there were no significant differences in UTUC-specific, bladder cancer-specific, and other causes of mortality between patients with advanced UTUC who received $\mathrm{ACH}$ and those who did not receive $\mathrm{ACH}$ after definitive surgery. Moreover, a multivariate competing risks regression model revealed that $\mathrm{ACH}$ was not an independent prognosticator in UTUC-specific, bladder cancer-specific, and other-cause death. Subgroup analysis also showed no significant differences between the observation and $\mathrm{ACH}$ groups, particularly among patients with stages pT34 and $\mathrm{pN}(-)$ and $\mathrm{pT}$ any and $\mathrm{pN}(+)$, according to competing risk of death. Conceptually, competing risk indicates an alternative outcome if a patient has the opportunity to experience one of several mutually exclusive events, and the occurrence of one event can prohibit the experience of any other event [17]. Because the conventional methods for survival analyses are not designed to reflecting the competing nature of different causes of mortality, these methods are not appropriate to accurately estimate the individual risk of the event of interest, such as mortality from unrelated causes [18]. For instance, patients with UTUC are at a risk of death from intravesical recurrence (or bladder cancer). Moreover, chemotherapy-related toxicity may compromise the survival outcomes in patients with renal impairment or older age, increasing other-cause mortality. In this regard, prognosis should be analyzed by considering this potential risk of bias. Therefore, our data based on a competing risk analysis, which is of more significant clinical importance, confirmed that postoperative chemotherapy could not improve the outcomes of the UTUC-, bladder cancer- and other cause-associated survival in patients with advanced UTUC.

Despite the lack of studies in patients with high-risk UTUC, Gandaglia and colleagues first presented the results of competing risk analysis for predicting disease-specific mortality, bladder cancer-related mortality, and other-cause mortalities according to age and stage in patients with localized UTUC who underwent RNU [19]. The authors reported that $18.1 \%(\mathrm{n}=1797), 31.2 \%(\mathrm{n}=3090)$, and $9.1 \%(\mathrm{n}=891)$ and $3090(31.2 \%)$ individuals died of UTUC-specific, bladder cancer-specific, and othercause mortalities, respectively [19]. These authors also found that age, tumor stage, female sex, type of surgery, grade, and tumor location were significantly associated with worse cancer-specific survival, and observed that ureteral tumor, stage, and tumor grade were related to bladder cancer-specific death [19]. Inman et al. [20] also reported that $46 \%$ of patients died owing to the competing risks by analyzing 168 patients with UTUC, and showed that preoperative predictors of cancer-specific mortality and competing mortality were invasive tumor characteristics $(\mathrm{HR}=$ 3.97; $\mathrm{P}<0.001)$ and older age $(\mathrm{HR}=1.07 ; \mathrm{P}<0.001)$. However, there are currently no studies based on competing risk analysis in patients with advanced stage UTUC. These studies can provide a better graphical tool for risk stratification of patients with UTUC undergoing RNU by estimating UTUC, bladder cancer and other-cause mortality, respectively, in the context of competing risk analysis. From a clinical standpoint, this novel approach to survival estimation can be useful for both clinicians and patients with UTUC in terms of initial counseling, decision-making, and surveillance planning after RNU.

We acknowledge that the present study is not devoid of limitations. First, as a retrospective data, this study has unavoidable drawbacks, such as selection bias especially who was suitable for cisplatin-based chemotherapy, and inconsistent data collection during the study period. Second, although comorbid disease, such as diabetes and chronic kidney disease can influence the long-term survival outcomes in patients who received systemic chemotherapy, there was a lack of detailed information regarding comorbid diseases in the population of the present study. Third, this study was performed by using single center data, and therefore; multicenter study should be conducted to validate our results and to provide more solid conclusions regarding the role of $\mathrm{ACH}$ in patients with locally advanced UTUC. Despite these disadvantages, our data highlight the ineffective impact of $\mathrm{ACH}$ on survival outcomes, especially based on competing risk analysis, in patients with advanced UTUC following RNU.

In summary, through competing risk analysis following propensity-score matching, we demonstrated that postoperative chemotherapy did not improve UTUC-specific and other causes-specific 
survival, in patients with locally advanced UTUC who underwent RNU. These results can offer practical information for clinicians regarding treatment decision making in these patients, who are at high risk of death due to competing causes.

\section{Supplementary Material}

Supplementary figures.

http://www.jcancer.org/v10p6896s1.pdf

\section{Acknowledgements}

This research was also supported by a grant of the Korea Health Technology R\&D Project through the Korea Health Industry Development Institute (KHIDI), funded by the Ministry of Health \& Welfare, Republic of Korea (HI17C0025). This study was also supported by Basic Science Research Program through the National Research Foundation (NRF) funded by the Ministry of Science and ICT (No.2018M3A9H10783360). The funders had no role in study design, data collection and analysis, decision to publish, or preparation of the manuscript.

\section{Competing Interests}

The authors have declared that no competing interest exists.

\section{References}

1. Leow JJ, Chong KT, Chang SL, et al. Upper tract urothelial carcinoma: a different disease entity in terms of management. ESMO Open. 2016; 1: e000126.

2. Roupret M, Babjuk M, Comperat E, et al. European Association of Urology Guidelines on Upper Urinary Tract Urothelial Cell Carcinoma: 2015 Update. Eur Urol. 2015; 68: 868-879.

3. Kim SP, Thompson RH. Challenges and changes for locally advanced upper tract urothelial carcinoma. Eur Urol. 2012; 62: 232-233.

4. Aziz A, Dobruch J, Hendricksen K, et al. Perioperative chemotherapy in upper tract urothelial carcinoma: a comprehensive review. World J Urol. 2017; 35: 1401-1407.

5. Sternberg CN, Skoneczna I, Kerst JM, et al. Immediate versus deferred chemotherapy after radical cystectomy in patients with pT3-pT4 or N+ M0 urothelial carcinoma of the bladder (EORTC 30994): an intergroup, open-label, randomised phase 3 trial. Lancet Oncol. 2015; 16: 76-86.

6. Leow JJ, Martin-Doyle W, Rajagopal PS, et al. Adjuvant chemotherapy for invasive bladder cancer: a 2013 updated systematic review and meta-analysis of randomized trials. Eur Urol. 2014; 66: 42-54.

7. Alfred Witjes J, Lebret T, Comperat EM, et al. Updated 2016 EAU Guidelines on Muscle-invasive and Metastatic Bladder Cancer. Eur Urol. 2017; 71: $462-475$

8. Kim HS, Lee JS, Jeong CW, et al. Adjuvant chemotherapy for locally advanced upper tract urothelial carcinoma: updated results of the Seoul National University Hospital experience. Int Braz J Urol. 2015; 41: 1067-1079.

9. Fujita $\mathrm{K}$, Taneishi $\mathrm{K}$, Inamoto $\mathrm{T}$, et al. Adjuvant chemotherapy improves survival of patients with high-risk upper urinary tract urothelial carcinoma: a propensity score-matched analysis. BMC Urol. 2017; 17: 110.

10. Necchi A, Lo Vullo S, Mariani L, et al. Adjuvant chemotherapy after radical nephroureterectomy does not improve survival in patients with upper tract urothelial carcinoma: a joint study by the European Association of Urology-Young Academic Urologists and the Upper Tract Urothelial Carcinoma Collaboration. BJU Int. 2018; 121: 252-259.

11. Seisen $T$, Krasnow $R E$, Bellmunt $J$, et al. Effectiveness of Adjuvant Chemotherapy After Radical Nephroureterectomy for Locally Advanced and/or Positive Regional Lymph Node Upper Tract Urothelial Carcinoma. J Clin Oncol. 2017; 35: 852-860.

12. Satagopan JM, Ben-Porat L, Berwick M, et al. A note on competing risks in survival data analysis. Br J Cancer. 2004; 91: 1229-1235.

13. Phe $\mathrm{V}$, Cussenot $\mathrm{O}$, Bitker $\mathrm{MO}$, et al. Does the surgical technique for management of the distal ureter influence the outcome after nephroureterectomy? BJU Int. 2011; 108: 130-138.
14. Leslie Sobin MG, Wittekind C. TNM classification of malignant tumours, 7th ed. Oxford: Wiley-Blackwell; 2009.

15. Epstein JI, Amin MB, Reuter VR, et al. The World Health Organization/International Society of Urological Pathology consensus classification of urothelial (transitional cell) neoplasms of the urinary bladder. Bladder Consensus Conference Committee. Am J Surg Pathol. 1998; 22: 1435-1448.

16. Roupret M, Babjuk M, Comperat E, et al. European Association of Urology Guidelines on Upper Urinary Tract Urothelial Carcinoma: 2017 Update. Eur Urol. 2018; 73: 111-122.

17. Kim HT. Cumulative incidence in competing risks data and competing risks regression analysis. Clin Cancer Res. 2007; 13: 559-565.

18. Berry SD, Ngo L, Samelson EJ, et al. Competing risk of death: an important consideration in studies of older adults. J Am Geriatr Soc. 2010; 58: 783-787.

19. Gandaglia G, Bianchi M, Trinh QD, et al. Survival after nephroureterectomy for upper tract urothelial carcinoma: a population-based competing-risks analysis. Int J Urol. 2014; 21: 249-256.

20. Inman BA, Tran VT, Fradet $\mathrm{Y}$, et al. Carcinoma of the upper urinary tract: predictors of survival and competing causes of mortality. Cancer. 2009; 115: 2853-2862. 